\title{
Bacterial Foraging Algorithm for Optimal Joint-Force Searching Strategy of Multi - SAR Vessels at Sea
}

\author{
Ngoc Ha Pham ${ }^{1}$, Minh Duc Nguyen² \\ 1 Hochiminh city University of Transport, No. 2, Vo Oanh Street, Ward 25, Binh Thanh District, Hochiminh \\ City, Vietnam; ha.pham@ut.edu.vn \\ 2 Vietnam Maritime University; 484 Lach Tray, Kenh Duong Ward, Le Chan District, Hai Phong city, \\ Vietnam; nguyenminhduc@vimaru.edu.vn \\ * Correspondence: ha.pham@ut.edu.vn
}

\begin{abstract}
Enhancing the effectiveness of search and rescue operation at sea is always a duty of utmost importance of the coastal states. The search area for distressed objects can be determined by using Monte Carlo simulation, combined with the Median-Filter. Once the search area has been identified, the success of search and rescue operations depends on the sweeping ability of search and rescue vessel at the probability area of the distress object with the minimum time. This is the important element to the success of the search and rescue operation as it minimizes the risk and cost for Search and rescue team. In this article, the authors study and propose the use of Bacterial Foraging Optimization Algorithm (BFOA) to calculate the optimal search and co-ordination route for many search and rescue vessels in Vietnam sea. The simulation results show that it is quite consistent with reality and BFOA can be effectively applied to determine a quick search.
\end{abstract}

Keywords: Search and rescue; optimal search algorithm; BFOA; multi-direction search; co-ordinate SAR operations.

\section{Introduction}

In the search and rescue (SAR) operation at sea, the minimum search time is the utmost important element to the success of the SAR operation as it minimizes the risks and costs for the SAR team. The success of a SAR mission often depends on the speed of identifying which operation is planned and carried out.

The drift of the distress object at sea is very complicated, as International Aeronautical and Maritime Search and Rescue (IAMSAR), Volume 2 [2]: "Two types of forces cause survival craft on the ocean to move or drift: wind and current. To compute the area where the survivors may be located, it is necessary to estimate the rate and direction of drift. This requires estimates of the winds and currents in and around the area containing the possible distress locations. The two main components causing the total drift (Drift) of the distress object is Leeway and Total Water Current. Monte Carlo simulation algorithm have been used to simulate the drifting trajectory of the distress object in real time then the Median-Filter was used to eliminate noise [7], [8]. Monte Carlo simulation calculated based on the wind and current, which has been tested for standard deviations to identify the area of search for distress objects (search area). Searching in a defined search area may be carried out by one or many search vessel and rescue (SAR) vessels. In [20], the authors have developed an adaptive Bacterial Foraging Optimization Algorithm (BOFA) to calculate the optimal multi-direction search route for a SAR vessel. In fact, the SAR operation is usually carried out by one or two SAR vessels. When two SAR vessels conduct the search, there must be a coordination and division of the search area for each SAR vessel to ensure the search effectiveness.

This paper proposes algorithm and applies it to compute the multi-direction search route for two search and rescue vessels coordinated on-scene in Vietnam sea. 


\section{Model for Route Planning Coordination of Two Search and Rescue Vessels}

The search operation for distress object in the search area can be divided into two periods:

- The stage of approaching the search area: it is considered the period when the SAR vessels moves from the standby position to the boundary of the search area, with the request of the shortest proceeding time.

- Search deployment stage in the region: Search-region is an area within which the probability of the target existence is larger than the limit value. The search must ensure that the total time the SAR vessel runs through the area where the maximum probability of drifting of the target is the smallest. This is a complex nonlinear problem because there are many different inputs, constantly changing over time, such as the influence of weather factors on victims, affecting the speed of SAR vessels when SAR vessels change direction ... and cannot be solved by analytic methods.

Developing the studies [8], [20], the author proposed an integrated model to calculate the optimal search coordination route for two SAR vessels considering the maneuverability characteristics of the SAR vessels

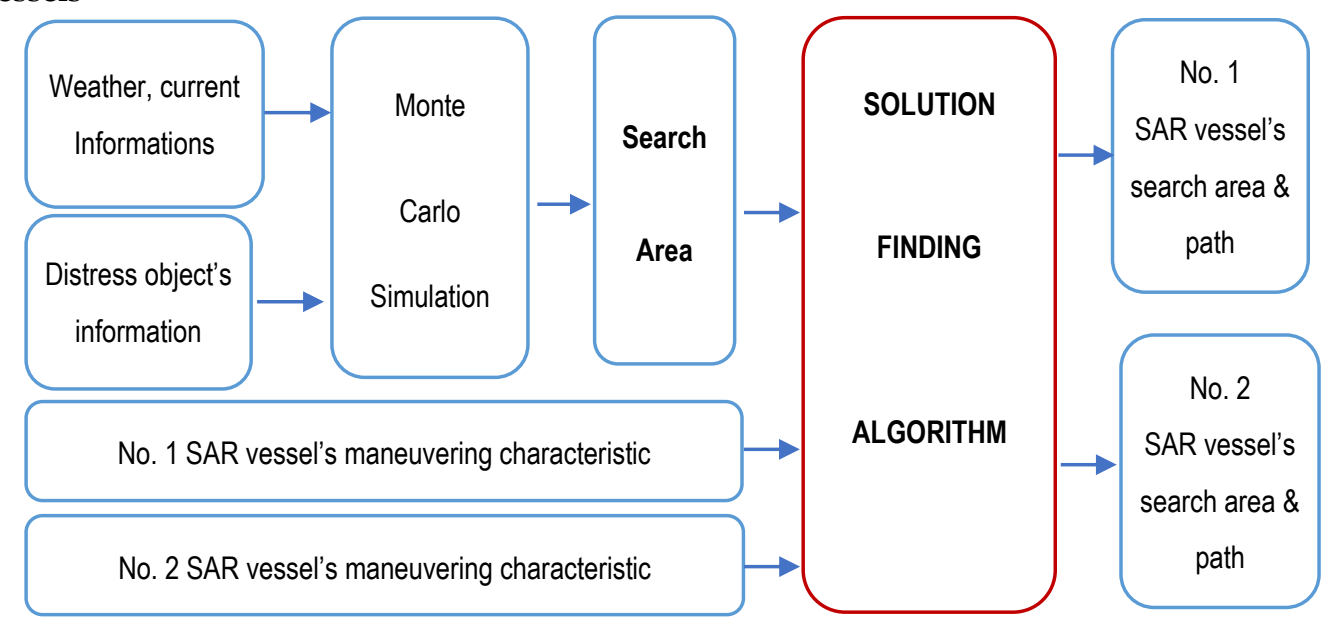

Figure 1. Optimization search route for two SAR vessels using BFOA

\section{Bacterial Foraging Optimization Algorithm Applying to the Multi-Direction Search Route for Two Search and Rescue Vessels}

\subsection{Bacterial Foraging Optimization Algorithm}

The bacterial foraging optimization algorithm (BFOA) is an advanced optimization technique introduced in 2002 by K.M. Passino, a professor of electrical engineering at the Ohio State University (USA) [6]. Within this technique, the method was originally proposed based on the social foraging behavior of the E.coli bacteria in the human intestine, on the way of searching for nutrients, a bacterium should make its foraging decision by considering two factors, namely energy consumption per unit of time and signal transduction with others. The BFOA was developed in order to provide a fast and optimal technique to solve the above problem. Since then, this technique has been successfully applied to many fields including optimal control engineering, network scheduling, image processing, etc [9] and has recently become one of the members of nature-inspired optimization algorithms [5]. However, it has not been applied to the SAR operation at the sea, especially at the Vietnam sea.

The BFOA, which was inspired by the bacteria forage over a landscape of nutrients, has been generally considered a promising solution for a variety of distributed optimization. The conventional BFOA consists of the following three steps:

1. Step 1 (Initialization): Initialize the population of the bacteria. Each bacterium is allocated randomly over the search space.

2. Step $\mathbf{2}$ (Evolution): Recursively manipulate the population to gradually develop by a three-step procedure: 
- Chemotaxis and swarming

- Reproduction

- Elimination and Dispersal

3. Step 3 (Termination): Return the most suitable solution so far, after a certain number of circles of generation evolution.

Thus, after each above circle, the bacterial individuals will be stronger than the previous ones, i.e., bacteria are getting closer to the optimal solution of the problem.

As mentioned in step 2, in nutrient foraging and evolving, bacteria population recursively goes through a process of 3 stages, namely the chemotaxis and swarming, reproduction, elimination and dispersal. The process enables the bacteria to gradually aggregate in the most favorable region or the region with the highest concentration of nutrient. Imitating foraging behavior of E.coli bacteria, BFOA seeks the best function in the search-space by conducting the local search through the bacteria chemotaxis, distributing local search by swarming, intensifying search in promising region by reproduction, and avoiding traps of local optimums by elimination and dispersal.

\subsection{Applying BFOA to plan co-ordinate search operations}

\subsubsection{The objective function}

The SAR operations at sea complies with the following principles:

- Coordinate, combine all resources to improve the efficiency of the SAR operations at sea, prioritize activities to rescue victims and protect the environment;

- Search planning, the SAR forces standing by for response activities by region, nature of the incident;

- Organizing timely receive and processing of distress information, prioritizing assurance of transducing information for response activities;

- The finding time of distressed object must be short;

- Areas which the probability of objects being drifted must be given priority for search;

- Ensuring safety for people and vehicles participating in SAR operations.

The problem considers the characteristics of SAR vessels (characteristics of speed, draft, fuel, operating time ...) and ensures the principles of SAR operations. The objective function of the search algorithm must ensure that the total time the SAR vessels sweep searches in the area that the highest probability of drifting to the object is the smallest. The objective function selected for two SAR vessels coordinating search is as follows:

$$
\text { Cost }=\operatorname{Max}\left[T T_{1}-W \times T P_{1}, T T_{2}-W \times T P_{2}\right]
$$

Determining the SAR route is towarded to the minimum Cost $\left(\right.$ Cost $=$ Cost $\left.t_{\text {min }}\right)$

- $\quad T_{1}$ (TotalTime1), $T_{2}$ (TotalTime2): the total amount of time SAR (No. 1 SAR vessel), SAR2 (No. 2 SAR vessel) vessels carrying out the SAR operation (with a certain speed) but less than the total time the vessel can operate (depending on fuel, weather ...)

- $\quad T P_{1}$ (TotalTimePropability1), $T P_{2}$ (TotalTimePropability2): Total of Probability of areas, which $\mathrm{SAR}_{1}$ vessel and $\mathrm{SAR}_{2}$ vessel swept in the search area;

- $\quad W$ : the coefficient (to give 2 compatible values, because Total Time Probability is too small compared to Total Time);

\subsubsection{General flowchart}

Each search strategy of the two SAR vessels is a bacterial individual in a set of bacteria which is considered a swarm. As a general rule of BFOA, optimal search based on bacterial colonies is carried out through steps shown on the below flowchart (Figure 2).

- Bacteria Position Initialization: generated randomly, each bacterium is allocated in a random creation of plans navigating two SAR vessels to search areas;

- Evolution: Implement population development loop: local adjustment of search plans, this step can be interpreted as using loops to adjust the search plan to the local optimal area, improve the 
search directions by a 3-step procedure (Chemotaxis and swarming, Reproduction, Elimination and Dispersal).

- Termination: Determining the most suitable bacterial position: After the loops have been implemented, the bacterial colonies have been converged enough and considered the optimal search result for two SAR vessels.

From sources of wind data and current data, which had been checked for standard deviations and the information of the distress object, the research [2], [6] used Monte Carlo simulation, combined with the Median-Filter (removes noise) to simulate the drifting trajectory of the distress object in real time, thereby identifying the search area. Based on the identified search area and the operating parameters of SAR vessels, this paper studies the application of the adapted BFO algorithm to build the optimal search co-ordination route for two SAR vessels.

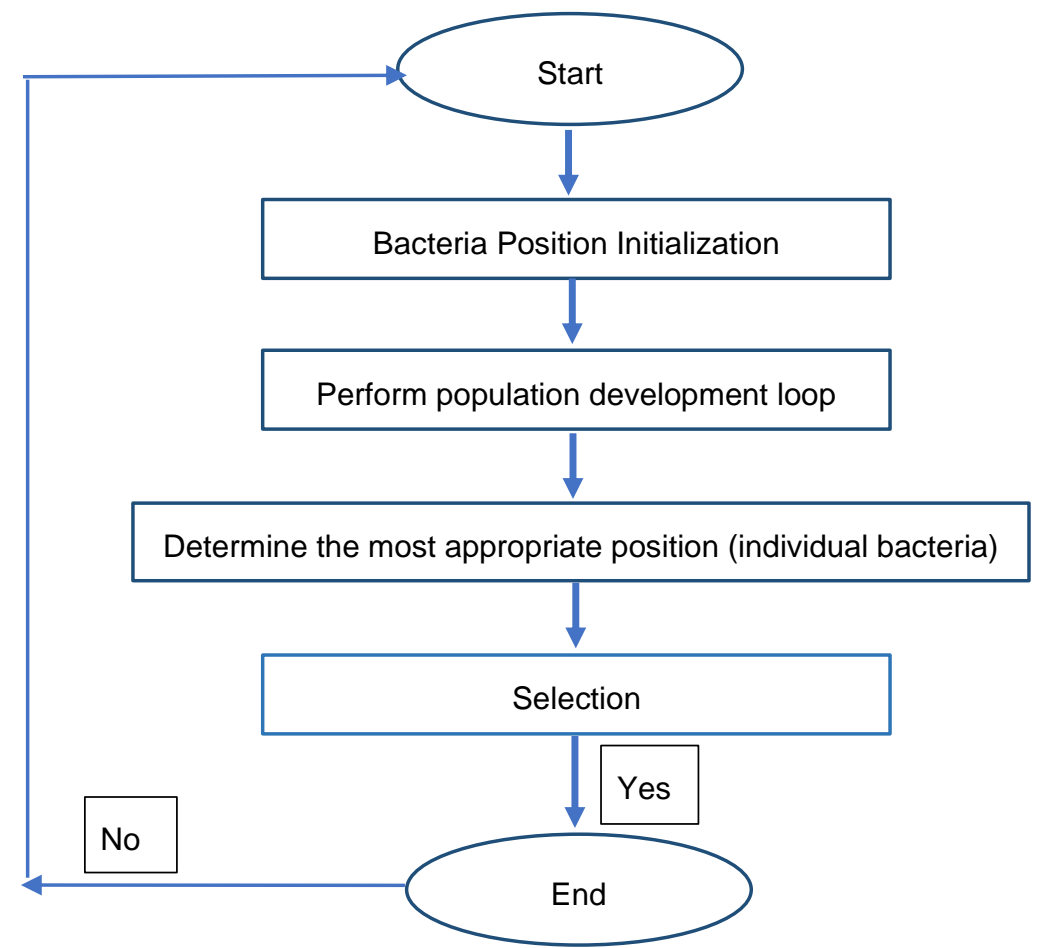

Figure 2. BFOA flowchart for calculating co-ordinate search route for two SAR vessels

\subsubsection{Loop development of bacteria generations}

\subsubsection{Initialize a bacterial populations}

Bacteria populations is a populations of initial random SAR plan. An individual bacterium is a separate rescue plan for two SAR vessels and takes the form:

- SepDir: Seperate Direction;

$$
\left\{\text { SepDir, SepRat, } V_{11}, V_{21}, V_{12}, V_{22}\right\}
$$

- SepRat: Seperate Rate;

- $V_{11}=\left\{W_{11}, W_{21} \ldots W P_{n 1}\right\}:$ direction to the search area for $\mathrm{SAR}_{1}$;

$-V_{21}=\left\{C_{11}, C_{21} \ldots C_{n 1}\right\}:$ sweeping direction in the search area for SAR 1 ;

$-V_{12}=\left\{W P_{12}, W_{22} \ldots W P_{n 2}\right\}$ : direction to the search area for $S_{2 A R}$;

$-V_{22}=\left\{C_{12}, C_{22} \ldots C_{n 2}\right\}:$ sweeping direction in the search area for SAR2.

\subsubsection{Evolution of individual bacteria}

Perform continuous loop evolution through bacterial generations. Improve the quality of individuals bacterial in 6 steps:

- Step 1: Select the SepDir randomly

- Replace: SepDir $=\operatorname{SepDir}+\delta$

- Check the new solution: 
$=>$ If this is the better solution, replace:

$$
\left\{\text { SepDir }_{\text {new }}, \text { SepRat, } V_{11}, V_{21}, V_{12}, V_{22}\right\}
$$

- Step 2: Select the SepRat randomly

$$
\text { SepDir }=\text { SepDir }+\delta
$$

- Replace: SepRat $=$ SepRat $+\delta$

- Check the new solution:

$=>$ If this is the better solution, replace:

$$
\left\{\text { SepDir, SepRat }{ }_{\text {new }}, V_{11}, V_{21}, V_{12}, V_{22}\right\}
$$

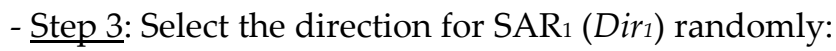

$$
\text { SepRat }=\text { SepRat }+\delta
$$

$$
\begin{aligned}
& W P_{i} \times L a t=W P_{i} \times L a t+\operatorname{Cos}\left(\operatorname{Dir}_{1}\right) \times \delta \\
& W P_{i} \times L \text { Lon }=W P_{i} \times \text { Lon }+\frac{\operatorname{Sin}\left(D i r_{1}\right) \times \delta}{\operatorname{Cos}(\text { Lat })}
\end{aligned}
$$

Check the new solution:

$$
\left\{\text { SepDir, SepRat, } V_{11 \text { new }}, V_{21}, V_{12}, V_{22}\right\}
$$

$\Rightarrow$ If this is the better solution, move $W P_{i}$ a distance $\Delta$ in the direction Dir1.

- Step 4: Select the $C i$ (sweeping direction for SAR 1 ) randomly:

- $\quad$ Replace $C_{i}$ with new direction:

- Check the new solution:

$$
C_{i}=C_{i}+\Delta
$$

$$
\left\{\text { SepDir, SepRat, } V_{11}, V_{21} \text { new }, V_{12}, V_{22}\right\}
$$

$=>$ If this is the better solution, replace:

$$
C_{i}=C_{i}+\Delta
$$

- $\underline{\text { Step 5: }}$ Select the direction for $\mathrm{SAR}_{2}\left(\operatorname{Dir}_{2}\right)$ randomly:

$$
\begin{aligned}
& W P_{i} \times L a t=W P_{i} \times L a t+\operatorname{Cos}\left(D_{i r}\right) \times \delta \\
& W P_{i} \times L o n=W P_{i} \times L o n+\frac{\operatorname{Sin}\left(D i r_{2}\right) \times \delta}{\operatorname{Cos}(\text { Lat })}
\end{aligned}
$$

Check the new solution:

$$
\left\{\text { SepDir, SepRat, } V_{11}, V_{21}, V_{12 \text { new }}, V_{22}\right\}
$$

$\Rightarrow$ If this is the better solution, move $W P_{i}$ a distance $\Delta$ in the direction Dir.

- Step 6: Select the $C i$ (sweeping direction for SAR2) randomly:

- $\quad$ Replace $C_{i}$ with new direction:

$$
C_{i}=C_{i}+\Delta
$$

- Check the new solution:

$=>$ If this is the better solution, replace:

$$
\left\{\text { SepDir, SepRat, } V_{11}, V_{21}, V_{12}, V_{22 \text { new }}\right\}
$$

$$
C_{i}=C_{i}+\Delta
$$

\subsubsection{Pairing and reproduction of populations}

- Randomly select individual father is a healthy bacterium in population: $\left\{V_{1 d a d}, V_{2 d a d}\right\}$;

- Randomly selecting the mother is a healthy bacterium in the population: $\left\{V_{1 m o m}, V_{2 m o m}\right\}$

- New born child has the form:

$$
\begin{gathered}
\left\{V_{1 \text { dad }}, V_{2 \text { dad-mom }}\right\} \\
V_{\text {2dad-mom }}=\left\{C_{1 \text { dad }}, C_{2 \text { dad }}, C_{3 m o m}, C_{4 m o m}\right\}
\end{gathered}
$$

\subsubsection{Elimination and Reproduction}

- Elimination: Eliminate weak bacterias (end of the list); 
- $\quad$ Reproduction: Create new random bacterias to replace discarded bacterias.

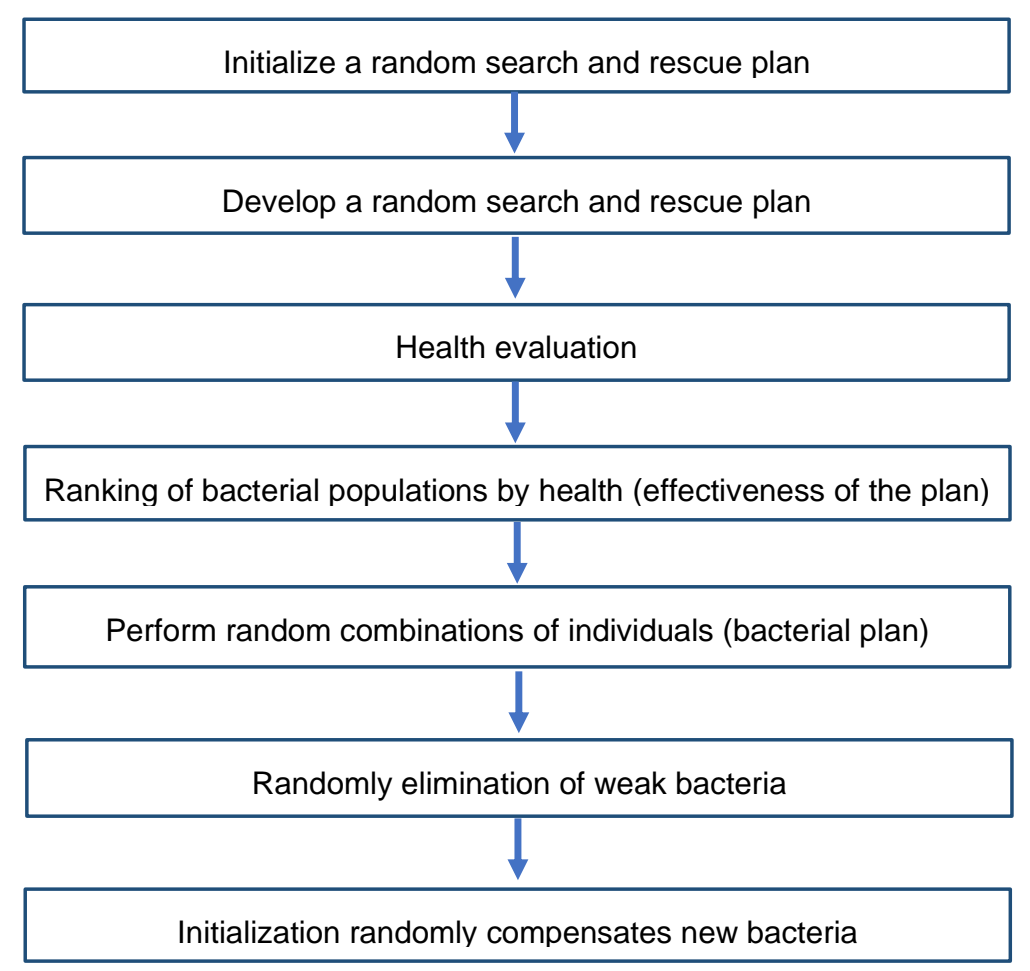

Figure 3. Develop loop bacteria over generations flowchart

\section{Simulation Results}

In the performance, a parallel sweep search was selected for the search route. Using software "SEARCH AND RESCUE SUPPORT SOFT" to simulate a co-ordination multi-directional search route for two SAR vessels for the below distress case:

- Distress object: Vietnam fishing vessel;

- Last Known Position (LKP): $11^{\circ} 00^{\prime} \mathrm{N} ; 109^{\circ} 00^{\prime} \mathrm{E}$;

- Time of LKP: 12h00, date: 1st August 2019;

- Standby position of SAR1: $08^{015^{\prime}} \mathrm{N} ; 106^{02} 0^{\prime} \mathrm{E}$;

- Standby position of SAR2: $12^{02} 1^{\prime} \mathrm{N} ; 109^{0} 36^{\prime} \mathrm{E}$.

- Start searching time: 20h00, date: $1^{\text {st }}$ August 2019

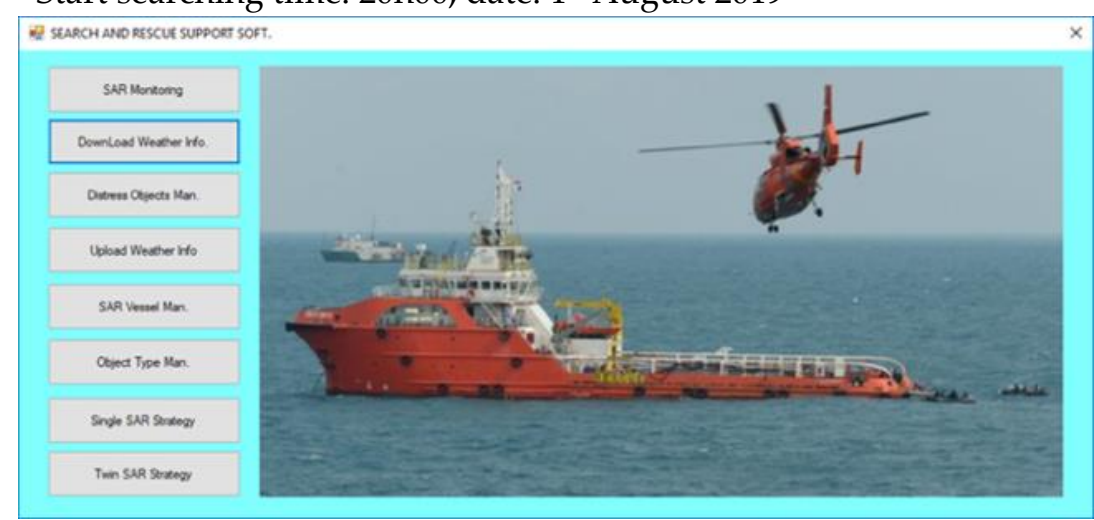

Figure 4. Software interface 


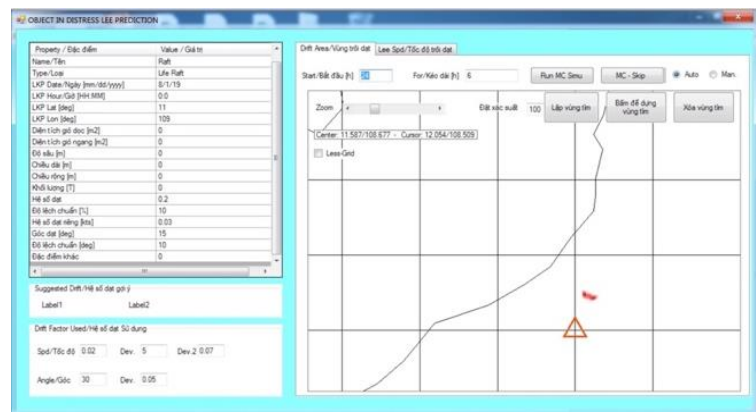

(a)

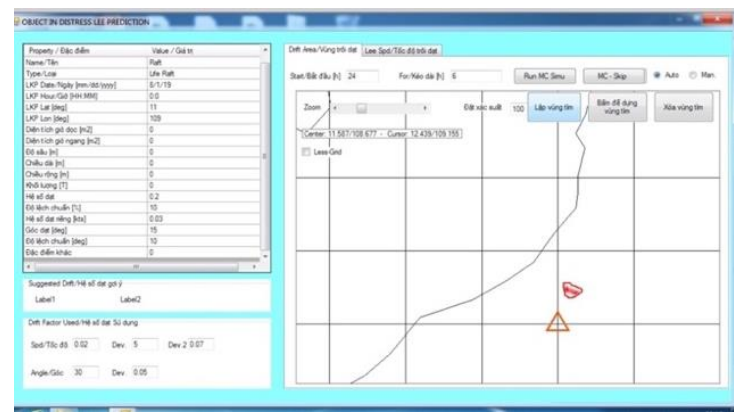

(b)

Figure 5. Simulation for drift of distress fishing vessel (a) drift area; (b) Search area

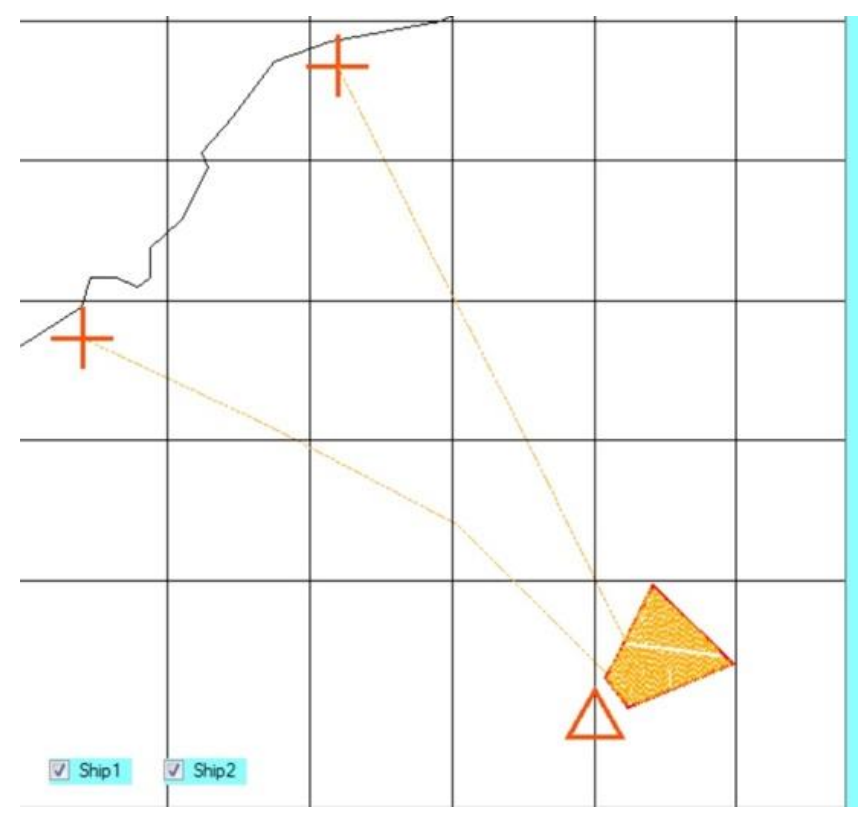

Figure 6. Multi-direction search plan for two SAR vessels

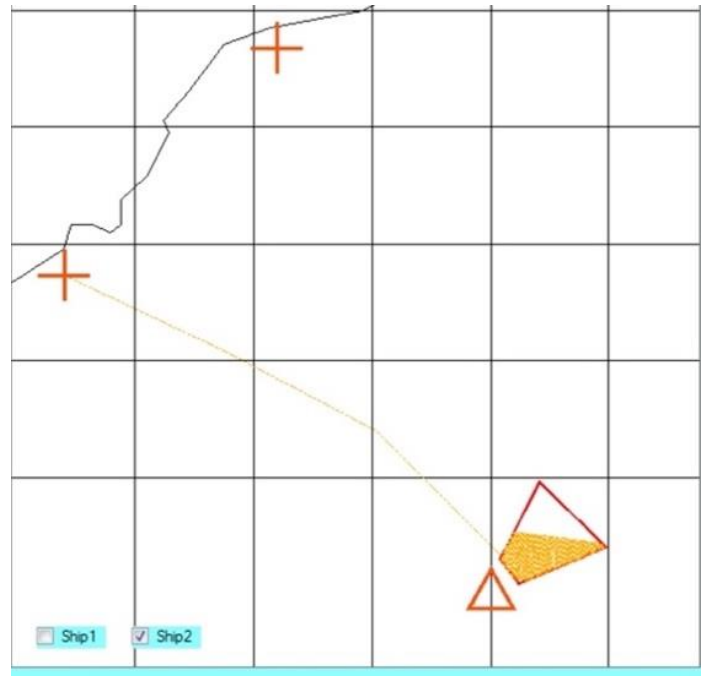

(a)

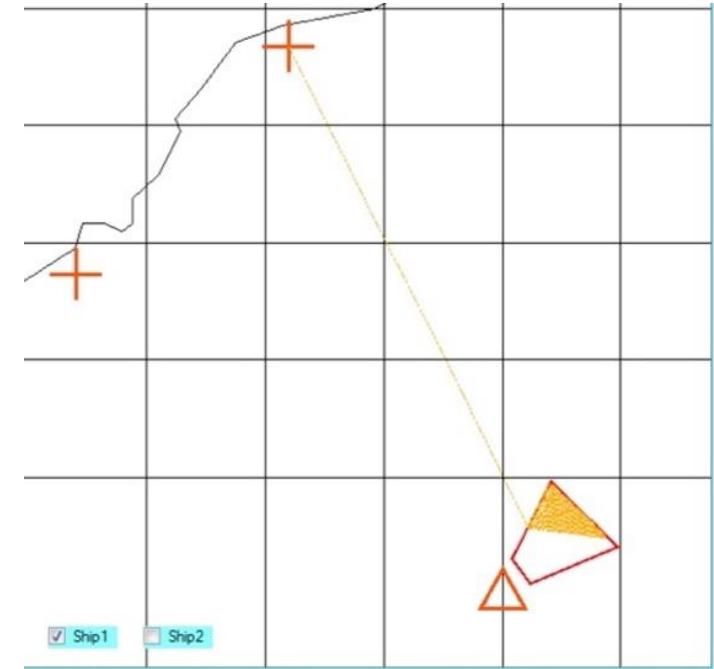

(b)

Figure 7. Multi-direction search for SAR vessels: (a) for SAR vessel No. 1; (b) for SAR vessel No. 2

\section{Conclusions}

The authors have developed an algorithm for optimal multi-direction search route in SAR operation for two SAR vessels strategy. Building an adapted BFO algorithm, can be used to calculate 
the optimal route for multi-directional search by two SAR vessels. It is help to reduce the searching time of distress object and increase chances of success in SAR operation at sea;

Monte Carlo simulation, combined with Median-Filter allows determining suitable search area. The BFO algorithm is highly reliable, able to calculate and give suggestions on the optimal search route even in cases of complicated changing weather conditions in Vietnam sea;

Building a computer program using BFO algorithm, the program is written appropriately, ensuring fast calculation time, so it can be applied in real conditions.

\section{References}

1. Allen A A, JV Plourde, “Review of Leeway: Field Experiments and Implementation," US Coast Guard Research and Development Center, Technical Report CG-D-08-99, 1999.

2. IMO (International Maritime Organization), International Aeronautical and Maritime Search and Rescue (IAMSAR), Volume 2, London: International Maritime Organization, 2016.

3. IMO (International Maritime Organization), International Aeronautical and Maritime Search and Rescue (IAMSAR), Volume 3, London: International Maritime Organization, 2016.

4. Minh Duc Nguyen, "A study on an integrated collision avoiding system for merchant ships," Doctor Thesis, Tokyo University of Marine Science and Technology, 2011.

5. Jason Brownlee. 2012. Clever Algorithms: Nature-Inspired Programming Recipes. [accessed 2018 Jun 20]. http://www.cleveralgorithms.com/nature-inspired/index.html.

6. KM Passino, "Biomimicry of bacterial foraging for distributed optimization and control," IEEE Control Systems Magazine, (Vol. 22), p.52-67, August 2002.

7. Ngoc Ha Pham, Minh Duc Nguyen, "Summary of weather information for forecasting object drift at sea in the search and rescue operation," Journal of Marine Science and Technology, Vietnam Maritime University, No 51, p.105-110, 8/2017.

8. Ngoc Ha Pham, Minh Duc Nguyen, “Weather Data Analysis and Drift Object Estimation by Monte Carlo Simulation for Vietnam's East Sea," in Proc. Asia Maritime \& Fisheries Universities Forum (AMFUF), 2017, p.467-477.

9. Vipul Sharma, SS Pattnaik, Tanuj Garg, "A Review of Bacterial Foraging Optimization and Its Applications," National Conference on Future Aspects of Artificial intelligence in Industrial Automation, in Proceedings of the NCFAAIIA, p 09-12, May 2012.

10. Alexey Bezgodov; Dmitrii Esin, "Complex Network Modeling For Maritime Search and Rescue Operations," Procedia Computer Science Volume 29, 2014, Pages 2325-2335;

11. C. Ying et al., "A Fast Bacterial Swarming Algorithm for high-dimensional function optimization", IEEE World Congress on Computational Intelligence, pp. 3135-3140, 2008

12. H. Chen, Y. Zhu, K. Hu, "Adaptive Bacterial Foraging Optimization", available at "http://www.hindawi.com/journals/aaa/2011/108269/"

13. M.D. Nguyen et al, Multi-Scale Automatic Route Planning Algorithms for Sea-Going Vessel, AMFUF 2013.

14. M.D. Nguyen et al, Automatic collision avoiding system for ship in congested water and at open sea, ICAIS 2012.

15. M.D. Nguyen et al, "A study on An Automatic Navigation System Basing on Radar and AIS data," World Congress 2009 - International Association of Institute of Naviation, 2009.

16. M. Tripathy, S. Mishra, et al., "Transmission loss reduction based on FACTS and bacteria foraging algorithm," Proceedings of the 9th International Conference on Parallel Problem Solving from Nature (PPSN '06), Vol. 4193 , pp. 222-231, 2006;

17. Liu Hongdan, et al., "Ship Collision Avoidance Path Planning Strategy Based on Quantum Bacterial Foraging Algorithm", Proceedings of the 2nd International Conference on Electrical, Computer Engineering and Electronics (ICECEE 2015) p.612-621;

18. S. Mishra, "A hybrid least square-fuzzy bacterial foraging strategy for harmonic estimation," IEEE Transactions on Evolutionary Computation, Vol. 9, No. 1, pp. 61-73, 2005

19. Y. Liu and K. M. Passino, "Biomimicry of social foraging bacteria for distributed optimization: Models, principles, and emergent behaviors," Journal of Optimization Theory and Applications, Vol. 115, pp. 603-628, 2002. 
20. Ngoc Ha Pham, Minh Duc Nguyen. An Evolutionary Algorithm for Optimal Multi-Direction Search Route in Search and Rescue Operation. International Journal on Advanced Science, Engineering and Information Technology, ISSN 2088-5334, Vol.9 (2019) No. 4, page 1199 - 1204;

21. Thomas M Kratzke et al, "Search and Rescue Optimal Planning System", online at https://ieeexplore.ieee.org/document/5712114. 